\title{
Percepções na saúde mental das gestantes durante a pandemia de Covid-19
}

\author{
Perceptions in the mental health of pregnant women during the Covid-19 pandemic \\ Percepciones en la salud mental de las embarazadas durante la pandemia Covid-19
}

Recebido: 25/11/2021 | Revisado: 30/11/2021 | Aceito: 04/12/2021 | Publicado: 05/12/2021

\author{
Misaele Silva Maciel \\ ORCID: https://orcid.org/0000-0002-8259-6578 \\ Centro Universitário FAMETRO, Brasil \\ E-mail: misaelesilva55@gmail.com \\ Valdirene Oliveira Avinte \\ ORCID: https://orcid.org/0000-0003-0198-7006 \\ Centro Universitário FAMETRO, Brasil \\ E-mail: valdireneavinte@outlook.com \\ Graciana de Souza Lopes \\ ORCID: https://orcid.org/0000-0003-3615-9040 \\ Centro Universitário FAMETRO, Brasil \\ E-mail: gracilopess@hotmail.com
}

\begin{abstract}
Resumo
Objetivo: identificar os principais agravamentos psicológicos ocasionados nas grávidas durante o isolamento da pandemia de Covid-19. Metodologia: trata-se de uma revisão integrativa da literatura (RIL), utilizando como a base de dados disponíveis na íntegra do Scielo, Pubmed e Lilacs. Posteriormente, foi realizado uma análise e um estudo qualitativo dos artigos encontrados, sendo selecionados apenas 13 artigos para compor a pesquisa, conforme as normas. Resultados: A saúde mental das gestantes foi bastante prejudicada na fase da pandemia, ocasionando ansiedade, medo, estresse, apreensão e sentimentos de solidão. Achados que foram observados em diferentes localidades no mundo. Observou-se significativa melhora em mulheres que tinham o apoio da família e que foram melhores observadas durante as suas consultas pelos profissionais. Conclusão: as adversidades psicológicas que as grávidas enfrentaram durante a pandemia que devem ser levados em consideração são: aumentando a qualidade da assistência prestada por todos os profissionais, gerando apoio social indispensável em épocas de crise.
\end{abstract}

Palavras-chave: Covid-19; Gestantes; Saúde mental; Impacto psicológico.

\begin{abstract}
Objective: to identify the main psychological aggravations caused by pregnant women during the isolation of the Covid-19 pandemic. Methodology: this is an integrative literature review (RIL), using as the database available in full Scielo, Pubmed and Lilacs. Subsequently, an analysis and a qualitative study of the articles found was carried out, with only 13 articles being selected to compose the research, according to the norms. Results: The mental health of pregnant women was significantly impaired during the pandemic phase, causing anxiety, fear, stress, apprehension and feelings of loneliness. Findings that have been observed in different locations around the world. There was a significant improvement in women who had family support and who were better observed during their consultations by professionals. Conclusion: the psychological adversities that pregnant women faced during the pandemic that must be taken into account are: increasing the quality of care provided by all professionals, generating essential social support in times of crisis.
\end{abstract}

Keywords: Covid-19; Pregnant women; Mental health; Psychological impact.

\section{Resumen}

Objetivo: identificar los principales agravamientos psicológicos causados por las mujeres embarazadas durante el aislamiento de la pandemia Covid-19. Metodología: se trata de una revisión integradora de la literatura (RIL), utilizando como base de datos disponible en su totalidad Scielo, Pubmed y Lilacs. Posteriormente, se realizó un análisis y un estudio cualitativo de los artículos encontrados, seleccionándose sólo 13 artículos para componer la investigación, de acuerdo con las normas. Resultados: La salud mental de las embarazadas se deterioró significativamente durante la fase pandémica, provocando ansiedad, miedo, estrés, aprensión y sentimientos de soledad. Hallazgos que se han observado en diferentes lugares del mundo. Hubo una mejora significativa en las mujeres que contaron con apoyo familiar y que fueron mejor observadas durante sus consultas por los profesionales. Conclusión: las adversidades psicológicas que enfrentaron las mujeres embarazadas durante la pandemia que se deben tomar en cuenta son: aumentar la calidad de la atención brindada por todos los profesionales, generando un apoyo social esencial en tiempos de crisis.

Palabras clave: Covid-19; Embarazadas; Salud mental; Impacto psicológico. 


\section{Introdução}

No dia 11 de março de 2020 a Organização Mundial de Saúde (OMS, 2020) fez um pronunciamento sobre os aumentos de casos que vinham ocorrendo, declarando que a Covid-19 era uma pandemia causada por um Coronavírus. Ele foi denominado como SARS-CoV-2, onde seu quadro clínico varia de uns simples sintomas de resfriado a um caso grave da doença, necessitando de um suporte ventilatório (Brasil, 2021).

Um dos maiores problemas do Coronavírus é a sua fácil transmissibilidade que acontece facilmente de pessoa para pessoa por um simples contato direto ou indiretamente. Por esse motivo que a doença se propagou tão facilmente em nível mundial. Em decorrência a isso, houve um aumento progressivo de depressão, ansiedade e até pensamentos de automutilação nas mulheres grávidas (Wu, et al., 2020).

As futuras mães vão precisar de uma atenção especial, por isso os cuidados Pré-natais têm essa importância de promover a saúde, prevenir, rastrear e diagnosticar determinadas doenças que afetam a saúde reprodutiva da mãe na geração do seu futuro filho, idealizando assim uma boa qualidade para todas as fases da gestação, constituindo-se em uma maternidade saudável (Brasil, 2021).

Os fatores que podem contribuir para uma gestação complicada, devido ao possível agravamento da Covid-19, são os riscos que as mesmas já podem apresentar como: idade avançada e estar acima do peso com grande massa corporal. Aliados a algumas comorbidades, como: a diabetes gestacional, hipertensão crônica e pré-eclâmpsia, podendo trazer desfechos desfavoráveis para ambos e aumentando a possibilidade de admissão na UTI (Allotey, et al., 2020).

Segundo Kassaw, Pandey (2020), a saúde mental das mães é primordial para um bom desenvolvimento da gravidez, porém, em decorrência da pandemia e das incertezas sobre o real impacto nelas, o estresse e a ansiedade foram aumentados. Gerando assim, o aumento das preocupações como seria o futuro de seus filhos, aumentando as suas aflições e inseguranças, gerando mais medo e podendo aumentar os riscos de depressão (Hamzehgardeshi, et al., 2021).

O período gestacional em si, já é algo estressante para a vida das mulheres e com a pandemia isso aumentou devido aos novos protocolos. A teleducação foi algo inovador que está possibilitando diminuir os níveis de estresse, angústia, ansiedade e até o medo. Dessa forma, a inclusão dessa ferramenta para elas serviu como um suporte de qualidade, gerando resultados positivos e diminuindo as preocupações (Derya, et al., 2021).

No entanto até a telessaúde tem suas limitações. Ela foi adotada em Nova York, por exemplo, após o avanço do Covid-19 para assim diminuir a transmissão no atendimento do pré-natal obstétrico, garantindo assim o cuidado continuado no conforto de casa. Mas essas tecnologias apresentam desconforto ou dificuldades técnicas com o seu uso e a necessidade de equipamentos hospitalares em casa, como o medidor de pressão arterial (Madden, et al., 2020).

A triagem universal foi outra proposta, mas que deve ser verificada individualmente, pois os altos ou baixos índices de pessoas infectadas ou prevalência da doença depende exclusivamente da área estudada. Dessa forma, em Madrid, eles observaram que uma conduta de testes sorológicos na admissão do parto serviria como uma estratégia de segurança tanto para os profissionais quanto para a família (Herraiz, et al., 2020).

As práticas preventivas são essenciais para a diminuição de uma doença, pois elas vão conseguir controlar a disseminação de um risco enfrentado. Porém os meios de informações para a população devem ser precisos e atualizados para não gerar mais caos, mais riscos psicológicos e podendo chegar até a morte com a depressão (Mariam, et al., 2021).

Dessa forma, durante a pandemia agravou-se tudo, incluindo a saúde mental das mães, pois tratou-se de uma situação de emergência, e elas já têm um risco de desenvolver transtornos mentais, afetando-as cerca de 20\% (OMS, 2020). Portanto, o objetivo deste trabalho é identificar os principais agravamentos psicológicos ocasionados nas grávidas durante o isolamento da pandemia de Covid-19. 


\section{Metodologia}

Trata-se de um estudo exploratório, descritivo, do tipo Revisão Integrativa de Literatura (RIL), é um método no qual sintetiza as pesquisas obtidas de modo ordenado e abrangente, fornecendo informações amplas sobre o conhecimento do problema abordado (Ercole et al., 2014).

Para a coleta de dados foram utilizadas as bibliotecas virtuais de saúde: Biblioteca Eletrônica Científica Online (Scielo), Pubmed e Literatura Latino-Americana e do Caribe em Ciências da Saúde (Lilacs), mediante aos descritores “COVID-19”, "Gestação" e "Saúde mental”.

Como os critérios de elegibilidade foram selecionados artigos originais, disponíveis gratuitamente, nos idiomas em português e inglês, publicados no período da pandemia de Covid-19, ou seja, entre os anos de 2020 a novembro de 2021 , que tratavam do tema pesquisado. E os critérios de inelegibilidade foram os artigos incompletos, outros idiomas que não fossem na língua inglesa ou portuguesa, publicações pagas, resumos, monografias, dissertações de mestrado e teses de doutorado.

Utilizando os descritores mencionados com o corretor “AND”, foram encontrados na sua totalidade 367 artigos, com a somatória das 3 bases de dados pesquisadas. Após os critérios de elegibilidade e inelegibilidade foi reduzido para 70 artigos, no qual foram lidos os resumos e selecionados apenas 13 que estavam condizentes com o objetivo da pesquisa. (Figura 1).

Figura 1: Fluxograma da escolha dos artigos.

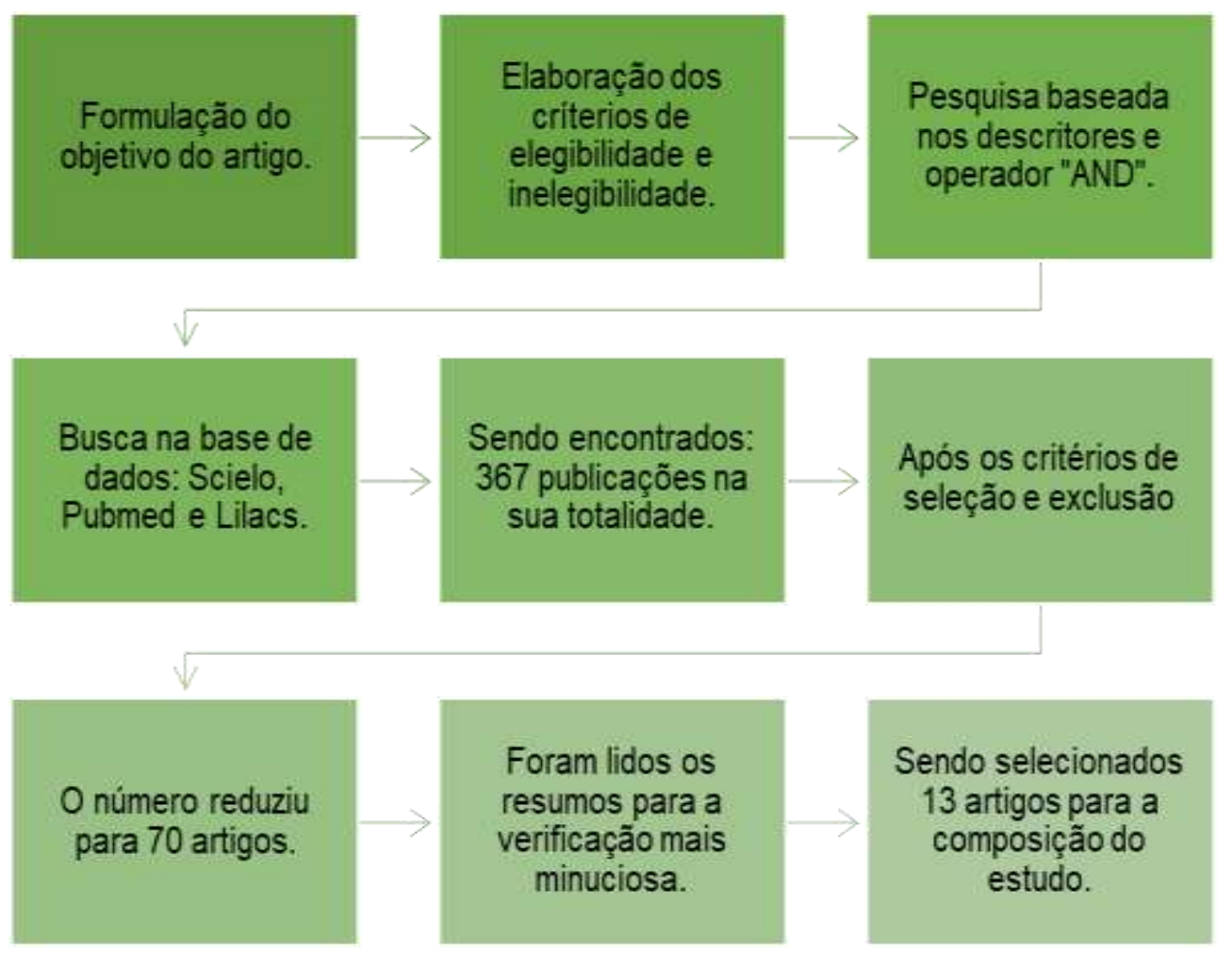

Fonte: Maciel, Avinte, Lopes (2021).

\section{Resultados}

Os 13 artigos selecionados foram analisados através dos critérios de elegibilidade a partir dos títulos obtidos, excluindo os artigos repetitivos, e posteriormente foi realizado uma análise, sendo lidos na íntegra. Após a análise e seleção dos artigos, eles foram colocados em forma de tabela para a melhor verificação e abrangência dos assuntos que abordavam. Mostrando assim, os pontos principais de cada autor, baseados em seus resultados. Dessa forma, eles foram organizados com o título, autores, ano e o resultado de cada estudo. (Tabela 1). 
Tabela 1: Estudos selecionados para a pesquisa.

\begin{tabular}{|c|c|c|c|}
\hline $\mathbf{N}^{\mathbf{0}}$ & TÍTULO & AUTORES (ANO) & RESULTADOS \\
\hline 1 & $\begin{array}{l}\text { Impacto psicológico da pandemia em } \\
\text { gestantes e puérperas brasileiras }\end{array}$ & Arrais, et al., 2021. & $\begin{array}{l}\text { Os principais temores foram as possíveis internações na UTI tanto } \\
\text { materna como neonatal, malformações no bebê, não poder } \\
\text { amamentar e não ter um acompanhante no parto. }\end{array}$ \\
\hline 2 & $\begin{array}{l}\text { Efeitos da pandemia de Coronavírus } 19 \text { na } \\
\text { ansiedade materna durante a gravidez: um } \\
\text { estudo observacional prospectivo }\end{array}$ & Mappa, et al.,2020. & $\begin{array}{l}\text { Na Itália } 178 \text { mulheres participaram desse estudo, no qual foi } \\
\text { demonstrado como o surto de COVID } 19 \text { e o bloqueio nacional } \\
\text { induziram um aumento significativo na ansiedade materna. }\end{array}$ \\
\hline 3 & $\begin{array}{l}\text { Percepções das mulheres sobre o COVID- } \\
19 \text { e suas experiências de saúde: uma } \\
\text { análise temática qualitativa de uma } \\
\text { pesquisa nacional com mulheres grávidas } \\
\text { no Reino Unido. }\end{array}$ & Karavadra, et al., 2020. & $\begin{array}{l}103 \text { mulheres participaram dessa pesquisa no Reino Unido e as } \\
\text { que tinham contato ou conheciam algum doente tiveram a } \\
\text { ansiedade aumentada. }\end{array}$ \\
\hline 4 & $\begin{array}{l}\text { Explorando os fatores relacionados ao } \\
\text { COVID-19 que influenciam a adoção de } \\
\text { serviços de atendimento pré-natal: um } \\
\text { estudo qualitativo entre mulheres em uma } \\
\text { comunidade rural no sudoeste da Etiópia }\end{array}$ & Hailemariam, et al., 2020. & $\begin{array}{l}\text { Na Etiópia } 44 \text { mulheres participaram desse estudo, mostrando } \\
\text { barreiras e dificuldades com o surto da pandemia, dentre eles estão } \\
\text { a má qualidade de atendimento e a ansiedade. }\end{array}$ \\
\hline 5 & $\begin{array}{l}\text { A ansiedade em relação à saúde prevê o } \\
\text { adiamento ou cancelamento de consultas } \\
\text { médicas de rotina entre mulheres em } \\
\text { estágio perinatal durante o bloqueio da } \\
\text { Covid-19 }\end{array}$ & Shayganfard, et al. 2020. & $\begin{array}{l}\text { As mulheres em estado perinatal tiveram mais ansiedade quando } \\
\text { existia alguma pessoa próxima com a doença, devido ao medo de } \\
\text { se infectar com o Covid-19. }\end{array}$ \\
\hline 6 & $\begin{array}{l}\text { Preocupações sobre transmissão, mudança } \\
\text { de serviços e local de nascimento no início } \\
\text { da pandemia COVID-19: uma pesquisa } \\
\text { nacional entre mulheres grávidas } \\
\text { dinamarquesas. }\end{array}$ & Schroder, et al., 2021. & $\begin{array}{l}\text { As mulheres dinamarquesas ficaram preocupadas com a } \\
\text { transmissão, mudanças na maternidade e restrição de parceiros. E } \\
\text { algumas sentiram tanto medo que planejaram o seu parto em casa, } \\
\text { com ou sem assistência obstétrica. }\end{array}$ \\
\hline 7 & $\begin{array}{l}\text { Covid-19 e saúde mental de gestantes no } \\
\text { Ceará, Brasil. }\end{array}$ & Machado, Correia., 2021. & $\begin{array}{l}\text { Quase metade das participantes apresentaram algum transtorno } \\
\text { mental elevado durante o distanciamento social, dentre eles estão } \\
\text { os sentimentos negativos em relação a pandemia de Covid-19. }\end{array}$ \\
\hline 8 & $\begin{array}{l}\text { Relação do medo de Covid-19 e qualidade } \\
\text { de vida relacionado à gravidez durante a } \\
\text { pandemia de Covid-19. }\end{array}$ & $\begin{array}{l}\text { Naghizadeh, Mirghafourvand., } \\
\text { 2021. }\end{array}$ & $\begin{array}{l}\text { No Irã, foi verificado que a relação do medo e qualidade de vida } \\
\text { afetaram negativamente as gestantes durante a Covid-19. }\end{array}$ \\
\hline 9 & $\begin{array}{l}\text { Um estudo piloto de métodos mistos de } \\
\text { risco perinatal e resiliência durante o } \\
\text { COVID-19. }\end{array}$ & Farewell, et al., 2020. & $\begin{array}{l}31 \text { mulheres responderam às perguntas, no qual tinham algumas } \\
\text { com sintomas de depressão alta, ansiedade moderada ou grave, } \\
\text { solidão e estresse. }\end{array}$ \\
\hline 10 & $\begin{array}{l}\text { Impacto do Coronavírus } 2019 \text { na saúde } \\
\text { mental e nas adaptações do estilo de vida de } \\
\text { mulheres grávidas nos Emirados Árabes } \\
\text { Unidos: um estudo transversal. }\end{array}$ & Hashim, et al. 2021. & $\begin{array}{l}\text { Foi demonstrado um estresse maior em gestantes que ficavam em } \\
\text { casa, outras sentiram medo e apreensão. Porém, algumas relataram } \\
\text { maior apoio familiar e tinham comportamentos de autocuidado. }\end{array}$ \\
\hline 11 & $\begin{array}{l}\text { A percepção de mulheres grávidas italianas } \\
\text { e novas mães sobre seu bem-estar } \\
\text { psicológico, estilo de vida, parto e } \\
\text { experiência de gerenciamento neonatal } \\
\text { durante o bloqueio da pandemia COVID- } \\
\text { 19: uma pesquisa baseada na web. }\end{array}$ & Stampini, et al., 2021. & $\begin{array}{l}\text { O impacto da pandemia ocasionou um escore alto para a ansiedade } \\
\text { e depressão. E durante o bloqueio, a prática de exercícios foi } \\
\text { diminuída, mas algumas relataram comer de forma mais saudável } \\
\text { nessa época. }\end{array}$ \\
\hline 12 & $\begin{array}{l}\text { Um estudo de métodos mistos avaliando o } \\
\text { impacto da pandemia de COVID-19 e } \\
\text { bloqueio no atendimento pré-natal de } \\
\text { mulheres grávidas em Puducherry, sul da } \\
\text { Índia }\end{array}$ & Ulaganeethi, et al., 2021. & $\begin{array}{l}\text { Na Índia } 150 \text { mulheres grávidas participaram do estudo. E elas } \\
\text { apresentaram algumas dificuldades devido a pandemia, dentre elas, } \\
\text { as dificuldades econômicas, falta de informação e estresse } \\
\text { psicológico. }\end{array}$ \\
\hline 13 & $\begin{array}{l}\text { A relação entre medo e ansiedade de } \\
\text { COVID-19, experiência de gravidez e } \\
\text { transtorno de saúde mental em mulheres } \\
\text { grávidas: um modelo de equação estrutural }\end{array}$ & Salehi, et al.,2020. & $\begin{array}{l}\text { A saúde mental das gestantes foi impactada diretamente através da } \\
\text { ansiedade e do medo, levando a resultados prejudiciais. }\end{array}$ \\
\hline
\end{tabular}

Fonte: Maciel, Avinte, Lopes (2021).

As revistas dos artigos selecionados foram: Brain Behav, Journal of Perinatal Medicine, J Prim Care Community Health (2), Family practice, Archives of psychiatric nursing, BMC gravidez e parto (4), DIAPHORA- Revista da sociedade de psicologia do Rio Grande do Sul, Int. J. Environ. Res. Public Health e Revista de Saúde Pública.

\section{Discussão}

\subsection{Categoria I: Principais agravamentos mentais observados em gestantes.}

Segundo Stampini et al. (2021), a ansiedade e a depressão foram os pontos mais observados nos agravamentos mentais das gestantes durante a pandemia. Segundo Farewell CV, et al (2020) No Colorado, as principais atribulações mentais 
foram a ansiedade moderada ou grave, depressão e sentimentos de solidão. Divergência com o achado de Hashim $M$ et al. (2021), no qual, nos Emirados Árabes Unidos, elas relataram o sentimento aumentado do medo, ansiedade, impotência e apreensão, mas que esse impacto foi algo leve.

Segundo Shayganfard et al. (2020), a ansiedade elevada prejudica a qualidade das emoções, devido a não deteç̧ão dos obstáculos enfrentados por este sentimento. No Irã as gestantes que tiveram mais ansiedade foram aquelas que tinham algum familiar doente, juntamente ao medo de se infectarem, fazendo com que elas adiassem ou até cancelassem as suas consultas médicas com o receio de exposição ao vírus. Os seus temores foram também em relação a ficar internada na UTI, perder o bebê, transmitir o Coronavírus verticalmente, não poder amamentar, o bebê adquirir alguma má formação e ficar na UTI neonatal (Arrais, et al., 2021).

Outro contratempo enfrentado foi o estresse, que estava presente, em consequência das incertezas que o isolamento acarretou, como o risco de exposição e a falta de redes de apoio (Farewell et al., 2020). E aliado a isso, Shayganfard et al. (2020), afirma que o estresse aumenta no pós-parto quando tem mais de uma criança na família.

Segundo Ulaganeethi et al. (2021), na Índia, o principal obstáculo enfrentado foi o medo, estando relacionado a fatores como: a mobilidade devido as leis impostas pelo o governo, falta de transporte e a falta de informação. E os contratempos psicossociais foram as dificuldades econômicas, falta de suporte social e o receio de ficarem doentes. Mesmo achado no Irã, no qual o medo estava presente, além disso, existia as preocupações acerca das consequências negativas que o vírus poderia ocasionar (Naghizadeh \& Mirghafourvand, 2021).

$\mathrm{Na}$ Itália, elas seguiram as restrições impostas pelo Governo, mas que também tinham medo de sair de casa e ir para o hospital, assim como, tinham receio de alguma anomalia prejudicar o seu filho, restringindo o crescimento fetal e o seu filho acabar nascendo prematuro (Mappa et al., 2020). Na Dinamarca, algumas ficaram com tanto medo que até consideraram o parto domiciliar com ou sem assistência obstétrica. Aliado, as aflições das mudanças na maternidade, limitações de atendimento e estarem sozinhas nas consultas (Schroeder, et al., 2021).

Segundo Karavrada et al. (2020), no Reino Unido, as participantes tinham inquietações acerca da influência da mídia, falta de apoio profissional, medo de morrer e tinham relutância em falar sobre as suas angústias por telefone. Recorrendo a internet para a obtenção de conselhos, pois, se sentiram desemparadas. Na Etiópia, as grávidas também tiveram má qualidade dos serviços, pois os profissionais as maltratavam (Hailemariam, et al., 2021).

Segundo Machado e Correia (2021), no Brasil, a falta de acompanhamento no pré-natal estava mais associada aos transtornos mentais, sendo agravada pelos sentimentos negativos em relação ao Covid-19. Explicados pelo Hailemariam et al. (2021), que afirma que durante a pandemia os serviços de atendimento no pré-natal foram prejudicados, por fatores como a superlotação de pacientes, múltiplos trabalhos dos profissionais e a falta de recursos.

Nesse contexto, os agravamentos enfrentados na saúde mental das gestantes, podem ter sido ocasionados por: mudanças nos setores, interrupção dos serviços, normas de segurança impostas pelo o governo e problemas financeiros, desencadeando as dificuldades psicoefetivas, como a ansiedade, medo, angústia, estresse e outros gatilhos emocionais.

\subsection{Categoria II: Fatores protetivos na saúde mental}

No Irã, a pesquisa mostrou que estar feliz tornou-se uma fonte de esperança, pois, melhorou a experiência durante a gravidez, diminuindo as adversidades na saúde mental ocasionados pela pandemia de Covid-19 (Salehi, et al., 2020).

Segundo Farewell, et al. (2020), o suporte emocional do parceiro, o aumento das plataformas de apoio e o autocuidado são os pontos primordiais no aumento da qualidade de vida. Corroborando com este achado, Naghizadeh e Mirghafourvand (2021), reafirmam que o apoio emocional da família é o ponto chave para elas não se sentirem sozinhas ou desamparadas, além disso, podem existir treinamentos adequados para os profissionais para o manejo do medo e a redução dos 
seus impactos.

Segundo Hashim, et al. (2021) e Stampini, et al. (2021), mostraram que durante a pandemia o apoio da família foi fundamental para elas conseguirem lidar com o isolamento, as gestantes que tiraram um tempo para descansar e que consumiram uma dieta balanceada acabaram melhorando o seu bem-estar mental. Porém, ainda existe a necessidade da priorização na saúde física durante esse período estressante.

Segundo Ulaganeethi, et al. (2021), durante essa fase de isolamento, a comunicação pode ser estabelecida pelo o uso de telefones celulares, ou seja, a telemedicina, podendo educar, ajudar e fornecer informações da Covid-19. E Karavrada, et al. (2020), reafirma que pode existir um foco maior em clínicas virtuais para a melhora da comunicação.

As clínicas podem aumentar a disponibilidade de acesso, logística, aumento de profissionais e suprimentos necessários na pandemia fazendo com que algumas unidades se adaptem melhor na pandemia (Hailemariam, et al., 2021). Oferecendo um atendimento para prevenir e reduzir as angustias, sendo uma intervenção que deveria ser prioridade para diminuir o sofrimento psicológico (Arrais, et al., 2021).

Nesse contexto, os profissionais precisam ter um conhecimento técnico-científico para conseguirem ajudar da melhor forma possível, em especial os enfermeiros, que estão presentes em todas as fases do processo saúde-doença. Aliado a isso, devem estar atentos às percepções das gestantes, ou seja, atender a todas as necessidades que essas mulheres apresentarem, embasado no cuidado da atenção individual.

\section{Conclusão}

As principais adversidades psicológicas ocasionadas nas grávidas durante o isolamento da pandemia de Covid-19 foram a ansiedade, solidão, medo, depressão, estresse e angústia. E foram explicados pelos temores de ficarem doentes, transmitir para os seus filhos, não estarem com acompanhante na hora do parto, serem mal recebidas na maternidade, dificuldades socioeconômicas que algumas passaram e por falta de informação.

As grávidas que se cuidaram mais, se alimentaram melhor e receberam apoio da família ou dos profissionais se sentiram menos sobrecarregadas, mostrando que algumas precisam apenas de mais atenção, em especial nessa fase que todos os seus hormônios estão alterados devido a gravidez.

Através desse estudo, foi possível compreender alguns sentimentos e preocupações que muitas grávidas sentiram no decorrer da pandemia. Assim, os profissionais precisam olhar mais atentamente para estas mulheres, pois a saúde mental sempre deve ser preservada. E verifica-se a necessidade de mais pesquisas sobre a temática, em relação a assistência que vai ser necessária para não prejudicar drasticamente a saúde mental das gestantes, visto que, ainda estamos enfrentando uma pandemia.

\section{Referências}

Allotey, J., Stallings, E., Bonet, M., Yap, M., Chatterjee, S., Kew, T., Debenham, L., Llavall, A. C., Dixit, A., Zhou, D., Balaji , R., Lee, S. I., Qiu, X., Yuan, M., Coomar, D., Sheikh, J., Lawson, H., Ansari, K., van Wely, M., \& van Leeuwen, E., (2020). Manifestações clínicas, fatores de risco e resultados maternos e perinatais da doença coronavírus 2019 na gravidez: revisão sistemática e meta-análise viva. BMJ (Clinical research ed.), 370, m3320.

Arrais, A., Amorim, B., Rocha, L., \& Haidar, A. C., (2021). Impacto psicológico da pandemia em gestantes e puérperas brasileiras. DIAPHORA- Revista da Sociedade de Psicologia do Rio Grande do Sul. 10(1).

Brasil, (2021). Coronavírus- Sobre a doença. Ministério da Saúde, from https://coronavirus.saude.gov.br/sobre-a-doenca.

Brasil, (2021). Importância do Pré-natal. Ministério da Saúde, from https://bvsms.saude.gov.br/dicas-em-saude/2198-importancia-do-pre-natal.

Derya, A. Y., Altiparmak, S., AkÇa, E., GÖkbulut, N., \& Yilmaz, A. N., (2021). Gravidez e planejamento do parto durante o COVID-19: Os efeitos da teleducação oferecida a mulheres grávidas sobre o sofrimento pré-natal e a ansiedade relacionada à gravidez. Obstetrícia, $92,102877$.

Ercole, F. F., Melo, L. S., \& Alcoforado, C. L. G. C., (2014). Revisão integrativa versus revisão sistemática. Revista Mineira de Enfermagem. 18.1. 
Farewell, C. V., Jewell, J., Walls, J., \& Leiferman, J. A., (2020). Um estudo piloto de métodos mistos de risco perinatal e resiliência durante o COVID-19. J Prim Care Community Health. 11: 2150132720944074.

Hailemariam, S., Agegnehu, W., \& Derese, M., (2021). Explorando COVID-19 Fatores Relacionados que Influenciam a Absorção de Serviços de Cuidados Pré-natais: Um Estudo Qualitativo entre Mulheres em uma Comunidade Rural no Sudoeste da Etiópia. J Prim Care Community Health. 12: 2150132721996892.

Hamzehgardeshi, Z., Omidvar, S., Amoli, A. A., \& Firouzbakht, M., (2021). Ansiedade relacionada à gravidez e seus fatores associados durante a pandemia de COVID-19 em mulheres grávidas iranianas: um estudo transversal baseado na web. BMC Pregnancy Childbirth, 21, 208.

Hashim, M., Coussa, A., Dhaheri, A. S. A., Marzouqi, A. A., Cheaib, S., Salame, A., Jamous, D. O. A., Naja, F., Hasan, H., Stojanobska, L., Mohamad, M. N., Bataineh, M. F., Faris, M. E., Daour, R. A., Obaid, R. S., Saleh, S. T., Osaili, T. M., \& Ismail, C., (2021). Impacto do Coronavírus 2019 na saúde mental e nas adaptações do estilo de vida de mulheres grávidas nos Emirados Árabes Unidos: um estudo transversal. BMC Pregnancy Childbirth, 21, 515.

Herraiz, I., Folgueira, D., Villalaín, C., Forcén, L., Delgado, R., \& Galindo, A., (2020). et al. Triagem universal para SARS-CoV-2 antes da admissão do parto durante a pandemia de Covid-19 em Madrid. Journal of Perinatal Medicine, 48 (9), 2020, 981-984.

Karavadra, B., Stockl, A., Snelling, E. P., Simpson, P., \& Morris, E., (2020). Percepções das mulheres sobre o COVID-19 e suas experiências de saúde: uma análise temática qualitativa de uma pesquisa nacional com mulheres grávidas no Reino Unido. BMC Pregnancy Childbirth, $20,600$.

Kassaw, C., \& Pandey, D., (2020). A prevalência de transtorno de ansiedade geral e seus fatores associados entre mulheres atendidas no serviço perinatal do hospital de referência da Dilla University, cidade de Dilla, Etiópia, abril de 2020 na pandemia de Covid. Heliyon. 6 (11): e05593.

Machado, M. M. T., \& Correia, L. L., (2021). Covid-19 e saúde mental de gestantes no Ceará, Brasil. Rev. Saúde Pública 55.

Madden, N., Emeruwa, U. N, Friedman, A. M., Aubey, J. J., Aziz, A., Baptiste, C. D., Coletta, J. M., D'Alton, M. E., Fuchs, K. M., Goffman, D., GyamfiBannerman, C., Kondragunta, S., Krenitsky, N., Miller, R. S., Nhan-Chang, C. L., Saint Jean, A. M., Shukla, H. P., Simpson, L. L., Spiegel, E. S., Yates, H. S., \& Ona, S., (2020). A adoção da telessaúde no atendimento pré-natal e nas atitudes do provedor durante a pandemia do COVID-19 na cidade de Nova York: uma análise quantitativa e qualitativa. American Journal of Perinatology, 37 (10) 1005-1014.

Mappa, I., Distefano, F. A., \& Rizzo, G., (2020). Efeitos da pandemia de coronavírus 19 na ansiedade materna durante a gravidez: um estudo observacional prospectivo. Journal of Perinatal Medicine. 48(6), 545-550.

Mariam, T. G., Kassie, B. A., Asratie, M. H., \& Abate, A. T., (2021). Os efeitos do medo e do conhecimento do COVID-19 na prática preventiva entre mulheres grávidas que frequentam cuidados pré-natais no noroeste da Etiópia, 2020: estudo transversal com base na instituição. Revista Internacional de Saúde da Mulher, 13 95-100.

Naghizadeh, S., \& Mirghafourvand, M (2021). Relação do medo de Covid-19 e qualidade de vida relacionado à gravidez durante a pandemia de Covid-19. Archives of Psychiatric Nursing. 35(4), 364-368.

Organização Mundial de Saúde, (2020). Saúde mental materna. from https://www.who.int/teams/mental-health-and-substance-use/maternal-mental-health.

Organização Mundial de Saúde, (2020). Discurso de abertura do Diretor-Geral da OMS no briefing para a mídia sobre COVID-19. 2020, from https://www.who.int/director-general/speeches/detail/who-director-general-s-opening-remarks-at-the-media-briefing-on-covid-19---11-march-2020.

Salehi, L., Rahimzadeh, M., Molaei, E., Zaheri, H., \& Esmaelzadeh-Saeieh, S., (2020). A relação entre medo e ansiedade de COVID-19, experiência de gravidez e transtorno de saúde mental em mulheres grávidas: Um modelo de equação estrutural. Cérebro e comportamento, 10 (11), e01835.

Schroder, K., Stokholm, L., Rubin, K. H., Jørgensen, J. S., Nohr, E. A., Petersen, L. K., \& Bliddal, M., (2021). Preocupações sobre transmissão, mudança de serviços e local de nascimento no início da pandemia COVID-19: uma pesquisa nacional entre mulheres grávidas dinamarquesas. BMC Pregnancy Childbirth 21,664 .

Shayganfard, M., Mahdavi, F., Haghighi, M., Sadeghi Bahmani, D., \& Brand, S., (2020). A ansiedade pela saúde prevê o adiamento ou cancelamento de consultas de rotina para atendimento médico de saúde entre mulheres em estágio perinatal durante o bloqueio da Covid-19. Jornal internacional de pesquisa ambiental e saúde pública, 17 (21), 8272.

Stampini, V., Monzani, A., Caristia, S., Ferrante, G., Gerbino, M., Pedrini, A., Amadori, R., Rabbone, I., \& Surico, D., (2021) A percepção de mulheres grávidas italianas e novas mães sobre seu bem-estar psicológico, estilo de vida, parto e experiência de gerenciamento neonatal durante o bloqueio da pandemia COVID-19: uma pesquisa baseada na web. BMC Pregnancy Childbirth 21, 473.

Ulaganeethi, R., Dorairajan, G., Ramaswamy, G., Thekkur, P., Olickal, J. J., Rajkumari, N., \& Saya, G. K., (2021) Eu estava com medo de acabar em outro aborto': um estudo de métodos mistos avaliando o impacto da pandemia de COVID-19 e bloqueio no atendimento pré-natal de mulheres grávidas em Puducherry, sul da Índia. Family Practice, 38, 23 - 29.

Wu, Y., Zhang, C., Liu, H., Chen, W., \& Dennis, C. L., (2020). Sintomas depressivos e de ansiedade perinatais em mulheres grávidas durante o surto da doença coronavírus em 2019 na China. American Journal of Obstetrics and Gynecology, $223,240$. 